\title{
Measles Outbreak Investigation in Pweto Health Zone-Haut-Katanga Province, DR of the Congo, 2018
}

\author{
Gabriel Kwete Woto Kwete ${ }^{1,2,3}$, Ekra Yao ${ }^{4}$, Jacques Kyungu Wa Numbi ${ }^{5}$ \\ ${ }^{1}$ Public Health Department, Official University of Mweka, Mweka, Kasaï, Democratic Republic of the Congo \\ ${ }^{2}$ Ministry of Health, EPI, Mweka, Kasaï, Democratic Republic of the Congo \\ ${ }^{3}$ WHO, Pweto, Haut-Katanga, Democratic Republic of the Congo \\ ${ }^{4}$ STOP Team Polio, CDC, Pweto, Haut-Katanga, Democratic Republic of the Congo \\ ${ }^{5}$ Pweto Health Zone Office, Pweto, Haut-Katanga, Democratic Republic of the Congo \\ Email: gabrielkwete@gmail.com
}

How to cite this paper: Kwete, G.K.W., Yao, E. and Numbi, J.K.W. (2020) Measles Outbreak Investigation in Pweto Health Zone-Haut-Katanga Province, DR of the Congo, 2018. Open Access Library Journal, 7: e6863.

https://doi.org/10.4236/oalib.1106863

Received: September 25, 2020

Accepted: November 20, 2020

Published: November 23, 2020

Copyright (C) 2020 by author(s) and Open Access Library Inc.

This work is licensed under the Creative Commons Attribution International License (CC BY 4.0).

http://creativecommons.org/licenses/by/4.0/

\begin{abstract}
Background: Sub-Saharan Africa reports repeated outbreaks of measles, a vaccine-preventable disease, which is notifiable under the Integrated Disease Surveillance and Response (IDSR) strategy in Democratic Republic of Congo (DRC). DRC has reported several outbreaks of measles in the last three years. Poor immunization coverage and weak health systems have been related with measles and other vaccination-preventable diseases' outbreaks. The DRC has committed to eliminating measles by 2020 . In this respect, we investigated one of the outbreaks that occurred in Pweto health zone (HZ), Haut-Katanga province, to describe the outbreak and identify risk factors. Methods: A cross-sectional study was designed to investigate a measles outbreak in Pweto health zone (HZ), Haut-Katanga province. Data entry and analysis were performed using EPI-Info version 7.1.0.6 and MS-Microsoft Excel. Findings: In seven months' time, four health areas affected by a measles outbreak in Pweto health zone reported 116-suspected cases and 13 deaths. The cumulative attack rate of $42.3 / 100,000$ population and case fatality rate (CFR) of $11.2 \%$ were recorded. Of these, $112(96.5 \%)$ cases were $\leq 15$ years of age, and 93 (80.2\%) were zero dose of measles vaccine. Although, all age groups were concerned, under five years old were the most affected with 84 cases $(72.4 \%)$. In response to the outbreak, a response immunization was organized with the support of MSF at the week 25 of the epidemic, when the epidemic curve started to decline. A response immunization involving 46,205 children from 6 to 59 was implemented and overall coverage of $99 \%$ (range: $95 \%$ - 105\%) was achieved. Case management with vitamin A supplementation, active case search, routine immunization improvement and health education were some
\end{abstract}


of the activities carried out to curb the outbreak. Conclusion: This confirmed measles outbreak was caused by failure to vaccinate ( $80.2 \%$ unvaccinated cases). Poor surveillance sensitivity and the community attitude about measles contributed to its spreading. Strengthening routine immunization, improving surveillance system and communication for behaviour change are required.

\section{Subject Areas}

Epidemiology, Infectious Diseases

\section{Keywords}

Measles, Outbreak Investigation, Outbreak Management, Pweto

\section{Background}

Measles is an acute, highly contagious viral disease caused by measles virus in the family Paramyxovirus, genus Morbillivirus, transmitted primarily by respiratory droplets or airborne spray from the coughs and sneezes of person infected with the disease to mucous membranes in the upper respiratory tract or the conjunctiva [1] [2].

Measles infection can result in serious complications such as blindness, encephalitis, or severe respiratory infections such as pneumonia [1] [2] [3]. The case fatality from measles is estimated to be $3 \%-5 \%$ in developing countries [1] [2].

Today, despite the availability of a safe, effective and relatively inexpensive vaccine for more than 40 years, measles still kills more than any other vaccine-preventable diseases among children. There is therefore the urgent need to revisit the measles immunization and vaccination program in Africa and in our country DRC with the sole aim of introducing a two-dose schedule to halt the endemic transmission [4].

The World Health Organization (WHO) member states have adopted the goal of measles elimination in five WHO regions, including Africa by 2020 [5]. Based on the experience of global eradication programmes, the success of disease elimination depends on the worst performing country [6]. One of the targets of measles elimination is vaccinating at least $95 \%$ of children in all districts with the first measles dose [7]. Priority interventions for the region are improving immunization coverage by strengthening routine and supplementary immunization activities targeting susceptible population groups. The DRC's Expanded Program on Immunization routine measles vaccination (EPI) includes one dose of measles vaccine administered to infants aged 9 - 11 months [8]. In 2010, WHO and UNICEF estimated that the EPI measles vaccine coverage among children younger than 12 months was only 68\% [9]. From 2011, a resurgence of measles cases has been reported from the country, with 133,802 cases in 2011 and 72,029 
in 2012. The world is witnessing an alarming surge in measles. In 2018, 229,068 measles cases were reported [10] [11]. According to WHO report, measles infections increased in all regions of the world in 2019. Global cases of measles in the first three months of 2019 have quadrupled compared with the same time last year; Africa had witnessed the most dramatic rise-up (700\%). In total, 170 countries reported 112,163 measles cases to WHO, in comparison to 28,124 cases across 163 countries during the same period in 2018 [12] [13]. The true figure may lie far above as measles is known to be heavily underreported. In low-income countries, poor access to vaccination, compounded by high birth rates, continues to produce massive epidemics. Large-scale outbreaks in Madagascar, DRC, Chad, Nigeria, Philippines, Yemen, and Ukraine fuelled the overall increase in incidence in all regions, the worst hit being the African region. DRC suffers from outbreaks recurring every 2 - 3 years, with peaks of 182,485 cases in 2005, 133,802 in 2011 and 88,381 cases in 2013. But the magnitude of the 2019 epidemic has been unprecedented with 311,471 reported cases, more than ever previously reported in the history of DRC surveillance. In mid-2018 a measles outbreak began in Katanga, eastern DRC, a region frequently experiencing measles epidemics. Early in 2019, the northeast was hit, and the outbreak further expanded to all provinces of the country. In 2018, $100 \mathrm{HZ}$ were declared in areas with confirmed epidemics, 124 more $\mathrm{HZ}$ were added in the first semester of 2019, and 129 in the next semester. Over two years $353 \mathrm{HZ}$ out of 519 had a confirmed epidemic.

In 2019, 6045 deaths due to measles were reported, mostly among children under 5 years of age. Poor access to health care and fatal complications occurring after the rash make many more deaths attributable to measles remain unnotified. In addition, measles puts children at high risk of acute malnutrition. Also, recent evidence tells us that beyond the direct immune suppression, measles resets the immune system re-exposing children to infections they were previously immune to [14]. The objective of this investigation is to assess the magnitude and identify the contributing factors for the measles outbreak in Pweto and generate evidence for the prevention and control of future outbreaks.

\section{Methods}

Study design: We conducted a cross-sectional study, including a review of the medical and epidemiological records and interviews in Pweto health zone, Province of Katanga, DRC from January 25 to August 5, 2018. Physicians, nurses, traditional healers and community agents were required to report suspected measles cases immediately, based on the criteria established by the Ministry of Health's $(\mathrm{MoH})$ Epidemiological Surveillance Division. Each case reported to the Health zone was followed up by a review of the case record and investigated by epidemiologists or nurses of the HZ team especially trained to confirm clinical diagnosis. In order to find additional cases, all health workers and community agents were instructed to ask patients about the presence of rash-like illness occurring in their 
neighbourhood as well as among school children.

To obtain informations on the outbreak, a line list and a semi-structured questionnaire were designed for the study. Demographic information was collected on patient characteristics, signs and symptoms of measles including date of onset, age, gender, date of visit to health facilities, setting of transmission, complications, outcome, and vaccination status. Only persons with a well-documented history of prior immunization were assumed to be vaccinated. All of them had received at least one live, attenuated measles vaccine dose.

Additional data were collected on cold chain, routine immunization and patient management in health facilities. We also conducted house-to-house and community active case searches and community-based surveillance for unreported cases of measles.

Study subjects: All persons affected by measles were subjects of the study.

Study Area: Pweto HZ is located in the south-eastern part of the DRC in Pweto territory. The population estimated to be 274,302 inhabitants in 2018, the DRC growth rate per annum is $3 \%$. The health zone has 16 health areas (HA) characterized by varying ecologies, climates, and populations. The areas are divided into 42 health posts. Administratively, the Pweto territory is divided into 2 HZs of Pweto and Kilwa. The total population of the territory is estimated to be 627,101 according to the 2018 census and housing projection.

Statistical analysis: Data were entered and analyzed using Epi-Info7 version 7.1.0.6 and Microsoft Excel. All data were cleaned for completeness before analysis. Attack rates, vaccination status, and case fatality rates were calculated and results were presented using graphs and tables.

Laboratory methods: Serum specimens were collected from twenty-two suspected cases and sent to the National Institute of Biomedical Research (INRB) in Kinshasa, where measles and rubella IgM ELISA are performed according to the global and national guidelines.

\section{Case definitions}

A suspected measles case: any person with generalized maculopapular rash and fever plus one of the following: a cough or coryza (a runny nose) or conjunctivitis (red eyes) [15].

A measles death: any death from an illness that occurs in a confirmed case or epidemiologically linked case of measles within one month of the onset of rash [11].

A confirmed measles case: a suspected case with laboratory confirmation (positive IgM antibody) or epidemiologically linked to confirmed cases [11].

Suspected measles outbreaks: defined as the occurrence of five suspected measles cases in one month in a defined geographic area such as a health zone, a health area or health facility catchment [15].

Confirmed measles outbreaks: defined as the presence of three or more laboratory-confirmed measles cases in a one-month time per health zone or health area or health facility [15].

A laboratory confirmed case: a suspected case, which has laboratory results, 
indicating infection (IgM positive or isolated for a measles virus) [15].

\section{Ethical Considerations}

This descriptive analysis used routine surveillance data collected by the HZ actors and WHO epidemiologists. Data were not nominative. During vaccine coverage surveys, verbal informed consent was obtained from each head of household visited. Privacy and confidentiality of patients were ensured by ensuring that no identifying information was recorded.

\section{Findings}

During the study period (January-August 2018) 183 patients out of a total of 185 notified cases were considered as having clinical measles after reviewing by the HZ of Pweto. The majority of suspected cases (116\% - 63.4\%) were investigated by epidemiologists or nurses of the $\mathrm{HZ}$ to collect information according to the questionnaire designed for this study. Only investigated cases were included in this analysis. Thirteen deaths were reported (Case Fatality Rate $(C F R)=11.2 \%)$ were reported from four health areas in Pweto health zone. Nineteen serum specimens were collected and 11 (57.9\%) were confirmed by laboratory investigation $(\mathrm{IgM}+)($ Table 1$)$ while the rest were epidemiologically linked and clinically compatible cases. The median age was 25.5 months ranging from 1 to 408 months. The interquartile range was 12 to 54 months ( 42 months) and there were $69(59.48 \%)$ females and $47(40.52 \%)$ males (Figure 1), but there was no evidence about the influence of the gender on the occurrence of the disease. Among the 116 cases, 84 (72.4\%) were $<5$ years old (Figure 2 ). The age-specific attack rates vary and were $135.84 / 100,000$ and $173.71 / 100,000$ populations for the age group $<1$ year and $1-4$ years respectively, while the overall attack rate was $42.24 / 100,000$ population. In total four health areas out of sixteen (25\%) were affected (Table 2) and the attack rate varies from health area to health area and ranged from 346 cases per 100,000 populations in Boma health area to 19 cases per 100,000 in Kamakanga (Table 3). The index case date onset was on January 25, 2018, and was reported from Boma health area (Kasanga quarter), in the crowded city of Pweto and seen at health facility level on February 6, 2018.

Many cases were reported in this health area because of the presence of qualified health actors ( $\mathrm{MoH}$ and partners) and the firm implication of political and community leaders in the outbreak management.

The cases started to build up slowly with a fluctuating trend and reached a peak on June 23-29, 2018 during the mass response immunization campaign to the cVDPV2 outbreak, which then started to decline shortly although it showed an inconsistent trend (Figure 3) when lucrative community agents were involved in the active search of cases.

The presence of weeks without reported cases reflects the failure of the surveillance system in some health areas. 
Regardless of its remoteness and difficulties in providing immunization service, the review of immunization coverage for the last 5 years indicates an overall coverage of $99 \%$, while it varied from health area to health area (range $=74 \%$ 138\%) (Table 3).

The high measles vaccination coverage in Boma contrasts with its high outbreak attack rate. The overall MCV immunization coverage in HA is very low, which is one of the epidemic risk factors. On the other hand of those affected children, 93 (80.2\%) of them were zero dose, while 23 (19.8\%) had received 1 - 4 MCV doses (Routine or Supplementary immunization dose) (Figure 4).

Interestingly of those unvaccinated cases, 66 (71\%) were $<5$ years old (Table 1$)$.

The presence of unvaccinated cases among adult population proves that the failure of the immunization system in the HZ is long-standing. The huge population movement in the crowded city of Pweto made it easy to extend the epidemic to neighbouring areas, that situation made it also difficult to provide routine immunization service. Despite the difficulties, an outbreak immunization response coupled to vitamin A administration was conducted starting on November 15, 2018, targeting 46,295 children from 6 to 59 months. Although, the disparity between health areas, the overall administrative coverage achieved was $111.6 \%$ (range $=94.7 \%$ to $163.3 \%)$ (Figure 5$)$.

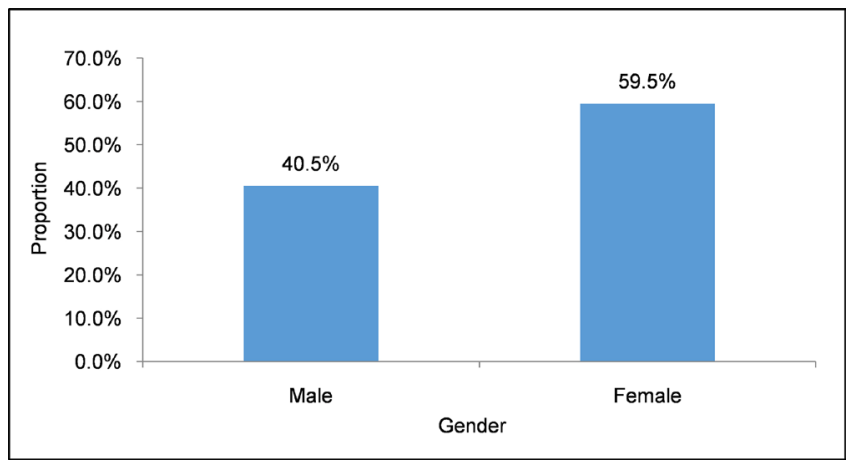

Figure 1. Distribution of measles cases by gender, Pweto HZ, 2018, DRC $(n=116)$. The female gender was the most affected.

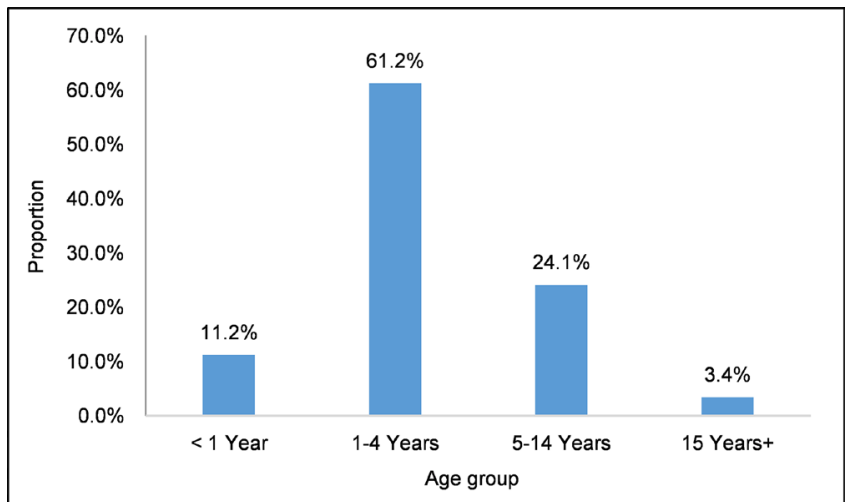

Figure 2. Distribution of measles cases by age groups, Pweto HZ, $2018(\mathrm{n}=116)$. The patients over one year old were the most affected. 
Table 1. Vaccination status of measles cases by age group, Pweto, DRC, $2018(\mathrm{n}=116)$.

\begin{tabular}{|c|c|c|c|c|c|c|c|}
\hline \multirow[b]{2}{*}{ Age } & \multicolumn{2}{|c|}{ Sex } & \multirow[b]{2}{*}{ Total } & \multicolumn{2}{|c|}{ Vaccination status } & \multirow{2}{*}{$\begin{array}{l}\text { Specimen } \\
\text { collected }\end{array}$} & \multirow[b]{2}{*}{ Result +} \\
\hline & Male & Female & & $\begin{array}{c}\text { Unvaccinated } \\
\mathrm{N}=93\end{array}$ & $\begin{array}{c}\text { Vaccinated } \\
(1 \text { dose }+) \mathrm{N}=23\end{array}$ & & \\
\hline$<1$ year & 8 & 5 & 13 & $36(38.7 \%)$ & $3(13 \%)$ & 3 & 1 \\
\hline $1-4$ years & 27 & 44 & 71 & $30(32.3 \%)$ & $11(47.8 \%)$ & 11 & 7 \\
\hline $5-14$ years & 1 & 27 & 28 & $10(10.8 \%)$ & $7(30.5 \%)$ & 4 & 2 \\
\hline 15 years + & 1 & 3 & 4 & $17(18.2 \%)$ & $2(8.7 \%)$ & 1 & 1 \\
\hline $\mathrm{HZ}$ & 37 & 79 & 116 & $93(100 \%)$ & $23(100 \%)$ & 19 & 11 \\
\hline
\end{tabular}

The presence of unvaccinated cases among the patients aged beyond one year is noted.

Table 2. Distribution of measles cases by health areas, Pweto HZ, $2018(\mathrm{n}=116)$.

\begin{tabular}{|c|c|c|c|c|}
\hline Health area & Case & Proportion (\%) & Death & CFR (\%) \\
\hline Boma & 103 & 88.8 & 9 & 8.7 \\
\hline Chanfubu & 5 & 4.3 & 2 & 40 \\
\hline Chalanshi & 5 & 4.3 & 1 & 20 \\
\hline Kamakanga & 3 & 2.6 & 1 & 33 \\
\hline $\mathrm{HZ}$ & 116 & 100 & 13 & 11.2 \\
\hline
\end{tabular}

Boma HA in the crowded city of Pweto reported a high number of measles cases.

Table 3. Measles administrative immunization coverage and attack rate by HA, Pweto HZ, 2014-2018, DRC.

\begin{tabular}{|c|c|c|c|c|c|c|c|}
\hline \multirow{2}{*}{ Health area } & \multicolumn{5}{|c|}{ Year } & \multirow{2}{*}{$\begin{array}{l}\text { Average }^{1} \\
\text { coverage }\end{array}$} & \multirow{2}{*}{ Attack rate ${ }^{2}$} \\
\hline & 2014 & 2015 & 2016 & 2017 & 2018 & & \\
\hline Boma & 111 & 113 & 96 & 117 & 108 & 109 & 346 \\
\hline Chalanshi & 91,3 & 95 & 96 & 120 & 95 & 100 & 22 \\
\hline Chamfubu & 103 & 34 & 150 & 55 & 114 & 91 & 25 \\
\hline Kakonona & 92 & 94 & 114 & 111 & 103 & 103 & $\mathrm{NA}^{3}$ \\
\hline Kamakanga & 82 & 87 & 87 & 96 & 100 & 90 & 19 \\
\hline Kapondo & 94 & 98 & 114 & 98 & 96 & 100 & $\mathrm{NA}^{3}$ \\
\hline Kapulo & 63 & 91 & 95 & 98 & 104 & 90 & $\mathrm{NA}^{3}$ \\
\hline Kasama & 96 & 99 & 94 & 86 & 90 & 93 & $\mathrm{NA}^{3}$ \\
\hline Kas-Kamulumbi & 67 & 55 & 85 & 76 & 86 & 74 & $\mathrm{NA}^{3}$ \\
\hline Katonta & 83 & 87 & 89 & 107 & 85 & 90 & $\mathrm{NA}^{3}$ \\
\hline Kinkalangu & 78 & 78 & 86 & 80 & 90 & 82 & $\mathrm{NA}^{3}$ \\
\hline Kizabi & 26 & 272 & 119 & 86 & 114 & 124 & $\mathrm{NA}^{3}$ \\
\hline Mumbalanga & 144 & 139 & 120 & 137 & 150 & 138 & $\mathrm{NA}^{3}$ \\
\hline Mwela & 101 & 93 & 98 & 101 & 103 & 99 & $\mathrm{NA}^{3}$ \\
\hline Mwenge & 77 & 95 & 89 & 89 & 92 & 88 & $\mathrm{NA}^{3}$ \\
\hline Sante & 118 & 117 & 100 & 81 & 101 & 103 & $\mathrm{NA}^{3}$ \\
\hline Health zone ${ }^{4}$ & 93 & 102 & 104 & 97 & 101 & 99 & 42.3 \\
\hline$\%$ of Health areas with MCV $\geq 95 \%{ }^{5}$ & 37.5 & 50 & 62.5 & 56.2 & 68.7 & 50 & \\
\hline
\end{tabular}

${ }^{1}$ Calculated by summing the five years' coverage and divided by five; target $95 \% ;{ }^{2}$ Attack rate/100,000 population in affected health area; ${ }^{3} \mathrm{Non}$ affected health areas; ${ }^{4}$ Calculated by dividing the number of MCV doses given by the target number of children $<12$ months of age and multiplying by 100 ; target $\geq 95 \%$; ${ }^{5}$ Calculated by dividing the number of health areas reporting $\geq 95 \% \mathrm{MCV}$ coverage by the number of health areas and multiplying by 100 ; target: $100 \%$; The high measles vaccination coverage in Boma contrasts with its high outbreak attack rate. The overall MCV immunization coverage in HA was very low. 


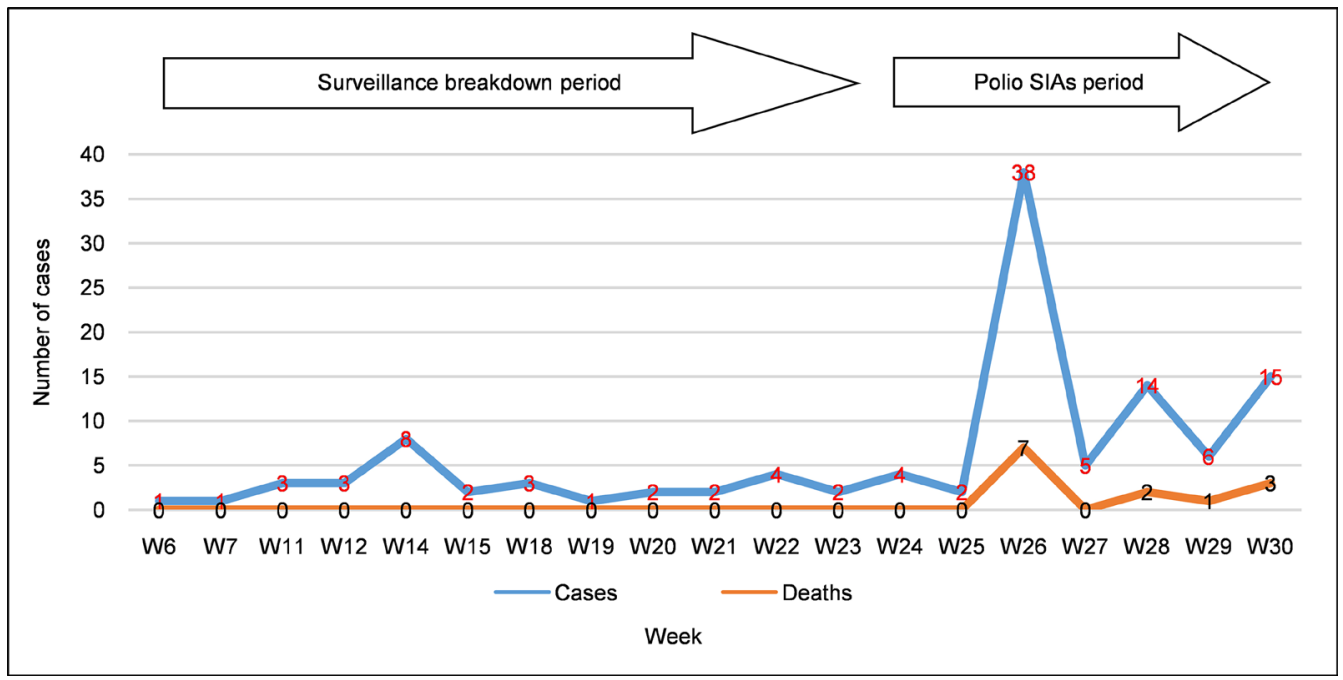

Figure 3. Measles Epi-curve by week of onset, Pweto HZ, DRC, August 2018 ( $\mathrm{n}=116)$. W: Epidemiological week. The presence of weeks without cases $(8,9,10,13,16$, and 17) was observed. The curve reached the peak during the cVDPV2 response immunization when lucrative community agents were involved in the active search of cases.

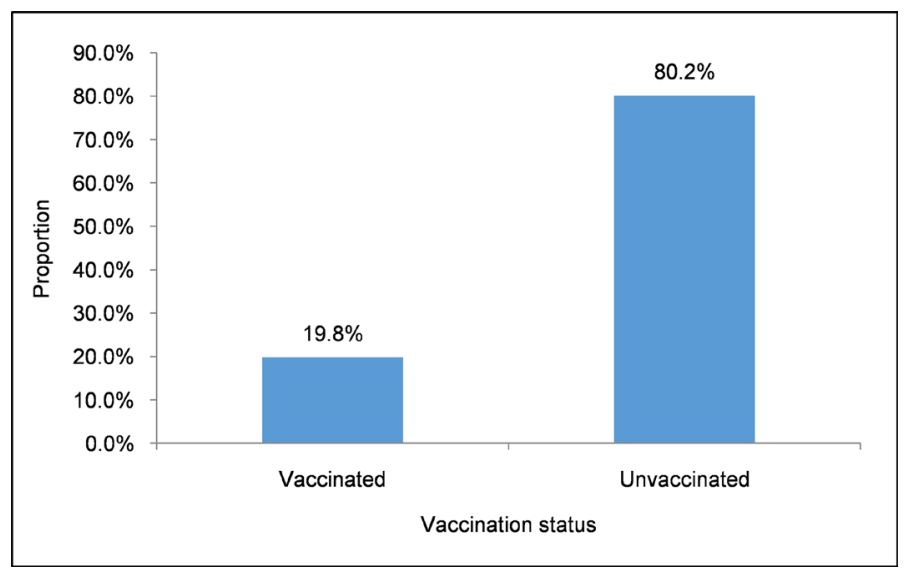

Figure 4. Vaccination status of measles cases, Pweto HZ, 2018, DRC $(\mathrm{n}=116)$. A huge proportion of unvaccinated cases was observed among the affected population.

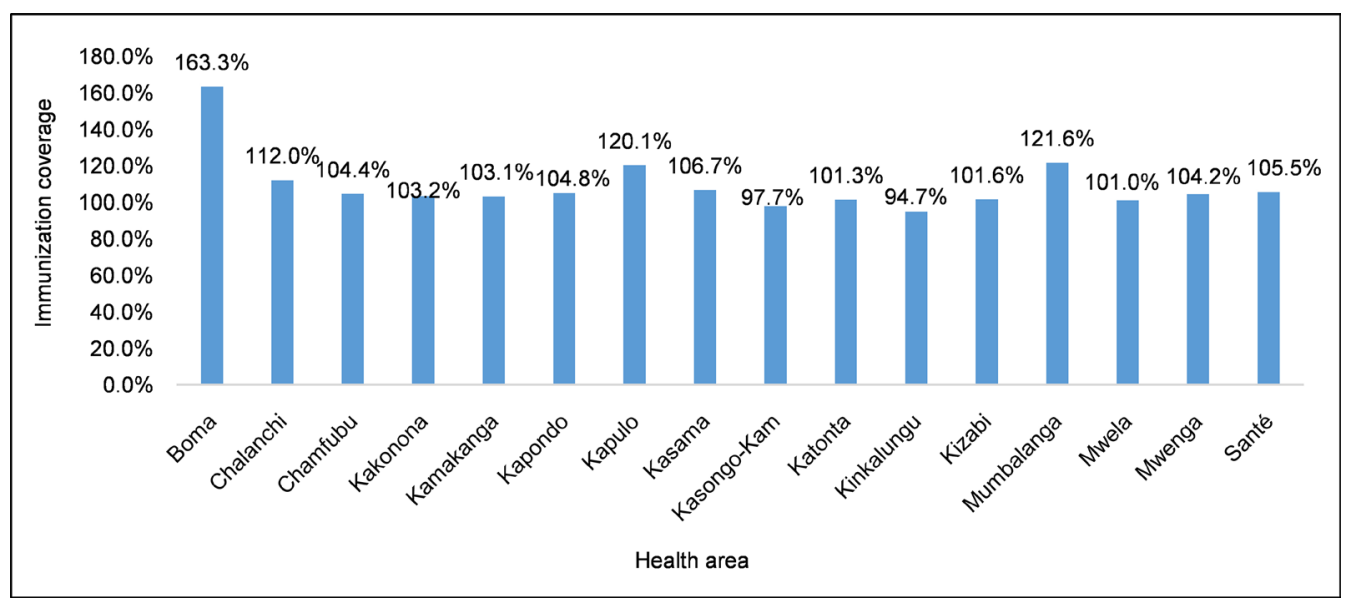

Figure 5. Immunization response coverage by health area, Pweto HZ, DRC, 2018. All health areas reached high immunization coverage $(\geq 95 \%)$. 


\section{Discussion}

The outbreak investigation showed that many cases of measles were unreported and the deaths reported do not reflect the reality considering the poor performance of the epidemiological system in the health district and the refractory behaviour of the population hiding affected patients. The female gender was more affected than the male (59.48\%), and more $<15$ years of age were primarily affected by the outbreak. We observed a high proportion of unvaccinated children (80.2\%) in this outbreak, indicating the low level of population immunity despite high coverage reported in the health zone (Table 3). The zone like the rest of the country had conducted catch-up campaigns for those who are aged between 6 59 months old with an interval of 3 - 4 years. The outbreak affected all age groups, while under five years children representing $71 \%$ were the most affected (Table 1 ). Close to the proportion published in a study about measles in the DRC (63\%) [16], even older than 15 years persons were affected, which may indicate the persistent low routine immunization coverage over several years and the accumulation of the susceptible population in the older age group that may have led to the current outbreak. Inadequate and poor cold chain management system coupled with hard to reach health areas makes it difficult to provide potent vaccine in routine immunization service likely also contributed to the outbreak. While the zone made some prominent progress in improving routine immunization, there is still pocket and hard to reach areas that are difficult to reach and provide routine immunization despite the introduction of routine immunization for over six decades. The proportion of unvaccinated patients in our findings $(80.17 \%)$ was higher than the one reported from Marshall Islands (59\%), Rio de Janeiro (72\% in $<1$ year old) [17] [18] and Ethiopia, in Guji zone (2017) (75\%) [15]. Nevertheless, the unvaccinated patients' proportion in this study is similar to the proportion reported in Ginnir district of Bale zone, Ethiopia in 2019 (79\%) [11]. We found a similar attack rate $(42 / 100,000)$ than the one recorded nationally during a measles outbreak, 41 per 100,000 populations, in 2008 [19]. We also found the disparity in the attack rate among health areas. This is due to the weakness of the surveillance system and the cultural misbehaviour of the community considering the measles a mysterious disease that has no medical treatment. That situation delayed detection and confirmation of the epidemic, which led to a delayed response. On the other hand, the higher attack rate may be due to the build-up of the susceptible population that may have contributed to the spread of the disease faster than expected. The disparity of attack rate may be the reflection of routine immunization as the performance of health areas concerning routine immunization is different between health areas and the epidemiological surveillance performance. The response vaccination conducted at the time of the decline of the Epi-curve may have had little or no impact on the overall epidemic control since many of the patients may have been already exposed or contracted the infection and may have recovered from measles illness. The highest attack rate (31 cases per 100,000) was observed among children $<5$ years old, which is the reflection 
of weak routine immunization in the affected health areas. This finding is similar to a study conducted in South Africa [20].

The attack rate in our finding $(42 / 100,000)$ is higher than the cumulative attack rate reported $(37 / 100,000)$ in South Africa's study and similar to the attack rates in other studies conducted including Shimada district $(41 / 100,000)$ in Ethiopia [20] [21].

Additionally, a relatively large proportion of cases $<9$ months were affected by the epidemic, which creates a concern regarding the age at which vaccination should start. This may be due to contributing factors like malnutrition or because children of this age group may have no sufficient maternal antibody at the very beginning which may indicate a long-standing problem with measles vaccination onset. The low proportion of affected health areas (25\%) associated with the peak of the EPI-Curve at the period of the cVDPV2 response campaign (week 26) proves that the community-based surveillance is not efficient and only the community agents engaged in the SIAs are involved in a lucrative active search of the affected children in the community. The case fatality rate in this study $(11.2 \%)$ was higher than the general measles case fatality in developing countries $(3 \%-5 \%)$ in general and up to $10 \%$ in closed outbreaks [22] when it is compounded by malnutrition [23]. The case fatality rate in this study is supposed to be very high because of the fact that death at the community level is not registered and this only includes health facility deaths. The study conducted in Simada district in Ethiopia indicated a higher CFR rate (13.4\%), including the study done on hospital admitted cases in Zimbabwe, Kenya, and Niger [24] [25]. However, our findings are also higher than that of Sudan (0.9\%) and Ethiopia (0, $2 \%$ in Guji zone of Oromia Region) [15]. To prevent measles outbreaks or interrupt transmission and to enhance elimination of measles, 95\% population immunity is needed. However, the administrative coverage of measles vaccination in the zone under review range from $83 \%-125 \%$ for the last four years with great disparity between health areas, which indicates suboptimal population immunity to prevent an outbreak. The absence of functional fridge at some health posts and hardship topography setup coupled with long travel distance to get the vaccine from the health centers may have affected the potency of the vaccine, and as a result, it could be the contributing factors for low population immunity while high coverage reported in some health areas where the epidemic occurred.

\section{Limitation of the Study}

The limitation of our study is the incompleteness of data and the inclusion of non-measles cases that do not fulfill the case definitions as line lists were collected by health workers at a lower level. That situation was also due to the weakness of the community-based surveillance in health areas. Additionally, death recorded only captures those deaths that occurred at health facility level, and community deaths were not reported. 
We also were unable to describe the epidemic profile and evaluate the impact of the vaccination after the implementation of the response immunization.

\section{Conclusions}

Almost all cases were reported by Boma, Chalanshi and Chanfubu health centers and in Katabe and Chanfubu hospitals in Pweto, the most populated city of the HZ. The persistent movement of the population and hard-to-reach areas challenged the routine immunization delivery system and during the investigation, it was witnessed that health facilities in some outbreak affected areas suffered from a lack of constant delivery of potent vaccine and regular maintenance of cold chain equipment. All of these HA have poor routine immunization data quality. Reaching every child (REC) strategy has to be strengthened in the HZ with particular emphasis to hard-to-reach areas to enhance the current immunization service.

Data quality self-assessment or cluster coverage survey should be conducted to verify the reported high vaccination coverage in some health areas (HA), especially in Boma. Periodic intensification of the routine immunization (PIRI) should be implemented to recuperate unvaccinated children under 23 months old. Surprisingly, the remote health areas did not report even one suspect measles case during the outbreak. The peak of the epidemic was observed when the cVDPV2 response immunization was implemented. During that period, AFP case notification and reporting were enhanced. We recommend that all potentially reporting sites should be exhaustively identified, prioritized and feasible strategies designed to be regularly supported for quality case detection, investigation and reporting. Private health facilities, traditional healers and prayer houses in the HZ must be targeted to strengthen the epidemiologic surveillance. Furthermore, a review of the case investigation tools to determine the contribution of traditional sites in case reporting would increase the evidence for further strengthening of the surveillance system at community level. Sensitization and advocacy have to be done to convince the communities and the political deciders to change the behaviour considering the measles as a mysterious disease without medical treatment. This way of thinking delays the detection of the epidemic, increases the attack and fatality rates and complicates the outbreak management. The community agents should not enhance the community-based surveillance only during lucrative immunization activities but also every time in order to strengthen the disease detection and forecast the outbreak profile. Realistically, should it also be possible to provide a motivation to the volunteer community agents involved in the vaccine-preventable diseases (VPDs) active search to expect better results. It is also proved that the first measles-containing vaccine (MCV) dose should be introduced into routine immunization programs before nine months of age seeing the occurrence of the disease before the single usual vaccination age ( 9 months).

The study shows that valuable information on the measles outbreak in very 
hard to reach and remote health areas is important to improve the completeness of the reports.

The study provides also information on the impact of early immunization response to control outbreaks of measles and the need to have sensitive and strong surveillance system to timely detect and respond to an outbreak of measles.

\section{Acknowledgements}

The authors acknowledge the contribution of all the health zone teams for the active part taken in the process of measles surveillance, particularly all surveillance officers working on detection, investigation, and outbreak management.

We are also grateful to the health workers, the community and the political leaders at various levels who directly or indirectly supported and contributed to the measles surveillance system and the elaboration of a contingency plan.

Finally, the authors gratefully thank MSF-F, CDC Atlanta and WHO for their technical and financial support and the DRC's EPI for vaccine and other materials supplying.

\section{Authors' Contributions}

All authors participated in the investigation, GKW designed and drafted the manuscript, critically reviewed, analysed and incorporated all comments. EY and JKN reviewed the manuscript and gave final approval for submitting for publication. Everybody read and approved the final version of the manuscript to be published.

\section{Conflicts of Interest}

The authors declare no conflicts of interest regarding the publication of this paper.

\section{References}

[1] Geremew, T.T., Gezie, L.D. and Abejie, A.N. (2019) Geographical Variation and Associated Factors of Childhood Measles Vaccination in Ethiopia: A Spatial and Multilevel Analysis. BMC Public Health 19, 1194.

https://doi.org/10.1186/s12889-019-7529-z

[2] World Health Organization Regional Office for Africa (2015) African Regional Guidelines for Measles and Rubella Surveillance. Geneva.

[3] World Health Organization (WHO) (1997) Guidelines for Epidemic Preparedness and Response to Measles Outbreaks. World Health Organization, Geneva.

[4] Okonko, I.O., Nkang, A.O., Udeze, A.O., Adedeji, A.O., Ejembi, J., Onoja, B.A., Garba, K.N., et al. (2013) Global Eradication of Measles: A Highly Contagious and Vaccine Preventable Disease-What Went Wrong in Africa? Journal of Cell and Animal Biology, 3, 119-140.

[5] World Health Organization (2012) Global Measles and Rubella Strategic Plan: 2012-2020. World Health Organization, Geneva.

http://www.who.int/immunization/newsroom/Measles_Rubella_StrategicPlan_2012 
2020.pdf

[6] Smith, R., Woodward, D., Acharya, A., Beaglehole, R. and Drager, N. (2004) Communicable Disease Control: A “Global Public Good” Perspective. Health Policy and Planning, 19, 271-278. https://doi.org/10.1093/heapol/czh032

[7] World Health Organization (2009) Measles Vaccines: WHO Position Paper. The Weekly Epidemiological Record, 35, 349-360.

[8] Ministry of Health of Democratic Republic of Congo (2012) Follow-Up Campaign against Measles, Action Plan 2010. Unpublished Results.

[9] World Health Organization African Region (2011) Measles Outbreaks and Progress towards Meeting Measles Pre-Elimination Goals. The Weekly Epidemiological Record, 13, 129-136.

[10] World Health Organization (2018) Measles Fact Sheet 2018. WHO, Geneva.

[11] Kalil, F.S., Gemeda, D.H., Bedaso, M.H. and Wario, S.K. (2020) Measles Outbreak Investigation in Ginnir District of Bale Zone, Oromia Region, Southeast Ethiopia, May 2019. The Pan African Medical Journal, 36, 20.

https://doi.org/10.11604/pamj.2020.36.20.21169

[12] World Health Organization (2019) Measles Fact Sheet: May 2019. WHO, Geneva.

[13] Center for Disease Control and Prevention (2019) Measles Update. CDC.

[14] Ducomble, T. and Gignoux, E. (2020) Learning from a Massive Epidemic: Measles in DRC. The Lancet Infectious Diseases, 20, 542. https://doi.org/10.1016/S1473-3099(20)30265-6

[15] Belda, K., Tegegne, A.A., Mersha, A.M., Bayenessagne, M.G., Hussein, I. and Bezabeh, B. (2017) Measles Outbreak Investigation in Guji Zone of Oromia Region, Ethiopia. The Pan African Medical Journal, 27, 9. https://doi.org/10.11604/pamj.supp.2017.27.2.10705

[16] Mancini, S., Coldiron, M.E., Ronsse, A., Ilunga, B.K., Porten, K. and Grais, R.F. (2014) Description of a Large Measles Epidemic in Democratic Republic of Congo, 2010-2013. Conflict and Health, 8, 9. https://doi.org/10.1186/1752-1505-8-9

[17] Hyde, T.B., Dayan, G.H., Langidrik, J.R., Nandy, R., Edwards, R., Briand, K., O'Leary, M.J., et al. (2006) Measles Outbreak in the Republic of the Marshall Islands, 2003. International Journal of Epidemiology, 35, 299-306. https://doi.org/10.1093/ije/dyi222

[18] de Oliveira, S.A., Soares, W.N., Dalston, M.O., de Almeida, M.T. and Costa, A.J. (1995) Clinical and Epidemiological Findings during a Measles Outbreak Occurring in a Population with a High Vaccination Coverage. Revista da Sociedade Brasileira de Medicina Tropical, 28, 339-343. https://doi.org/10.1590/S0037-86821995000400006

[19] EHNRI, Federal Democratic Republic of Ethiopia (2012) Guideline on Measles Surveillance and Outbreak Management. Addis Ababa.

[20] Ntshoe, G.M., McAnerney, J.M., Archer, B.N., Smit, S.B., Harris, B.N., Tempia, S., Blumberg, L.H., et al. (2013) Measles Outbreak in South Africa: Epidemiology of Laboratory-Confirmed Measles Cases and Assessment of Intervention, 2009-2011. PLoS ONE, 8, e55682. https://doi.org/10.1371/journal.pone.0055682

[21] Aragaw, M. and Tilay, T. (2012) Measles Outbreak in Simada District, South Gondar Zone, Amhara Region, May-June 2009: Immediate Need for Strengthened Routine and Supplemental Immunization Activities (SIAs). Ethiopian Journal of Health Development, 26, 115-118.

[22] Mandomando, I., Naniche, D., Pasetti, M.F., Cuberos, L., Sanz, S., Vallès, X., Levine, 
M.M., et al. (2011) Assessment of the Epidemiology and Burden of Measles in Southern Mozambique. The American Journal of Tropical Medicine and Hygiene, 85, 146-151. https://doi.org/10.4269/ajtmh.2011.10-0517

[23] Rice, A.L., Sacco, L., Hyder, A. and Black, R.E. (2000) Malnutrition as an Underlying Cause of Childhood Deaths Associated with Infectious Diseases in Developing Countries. Bulletin of the World Health Organization, 78, 1207-1221.

[24] Kambarami, R.A., Nathoo, K.J., Nkrumah, F.K. and Pirie, D.J. (1991) Measles Epidemic in Harare, Zimbabwe, despite High Measles Immunization Coverage Rates. Bulletin of the World Health Organization, 69, 213.

[25] Nandy, R., Handzel, T., Zaneidou, M., Biey, J., Coddy, R.Z., Perry, R. and Cairns, L. (2006) Case-Fatality Rate during a Measles Outbreak in Eastern Niger in 2003. Clinical Infectious Diseases, 42, 322-328. https://doi.org/10.1086/499240

\section{Abbreviations}

AFP: Acute Flaccid Paralysis

AR: Attack Rate

CDC: Centers for Disease Control and Prevention

CFR: Case Fatality Rate

cVDPV2: Circulating Vaccine-Derived Poliovirus Type 2

DRC: Democratic Republic of Congo

ELISA: Enzyme-Linked Immunosorbent Assay

EPI: Expanded Program on Immunization

HA: Health Area

HZ: Health Zone

IDSR: Integrated Disease Surveillance and Response

IgM: Immunoglobulin $M$

INRB: Institut National de Recherche Biomédicale (National Institute of Biomedical Research)

MCV: Measles-Containing Vaccine

MoH: Ministry of Health

MSF: Médecins Sans Frontières

PIRI: Periodic Intensification of Routine Immunization

REC: Reaching Every Child

SIA: Supplementary Immunization Activity

UNICEF: United Nations Children's Fund

VPD: Vaccine-Preventable Disease

WHO: World Health Organization. 